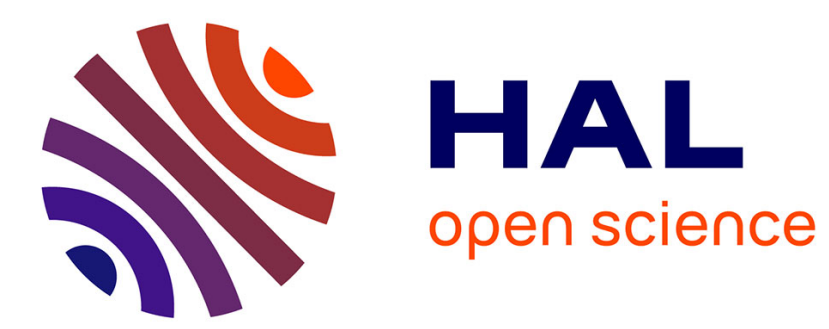

\title{
Approximate transmission conditions through a weakly oscillating thin layer \\ Clair Poignard
}

\section{To cite this version:}

Clair Poignard. Approximate transmission conditions through a weakly oscillating thin layer. Mathematical Methods in the Applied Sciences, 2009, 32 (4), pp.435-453. 10.1002/mma.1045 . inria00334772

\section{HAL Id: inria-00334772 \\ https://hal.inria.fr/inria-00334772}

Submitted on 27 Oct 2008

HAL is a multi-disciplinary open access archive for the deposit and dissemination of scientific research documents, whether they are published or not. The documents may come from teaching and research institutions in France or abroad, or from public or private research centers.
L'archive ouverte pluridisciplinaire HAL, est destinée au dépôt et à la diffusion de documents scientifiques de niveau recherche, publiés ou non, émanant des établissements d'enseignement et de recherche français ou étrangers, des laboratoires publics ou privés. 


\title{
APPROXIMATE TRANSMISSION CONDITIONS THROUGH A WEAKLY OSCILLATING THIN LAYER
}

\author{
CLAIR POIGNARD
}

\begin{abstract}
We study the behavior of the electro-quasistatic voltage potentials in a material composed by a bidimensional medium surrounded by a weakly oscillating thin layer and embedded in an ambient medium. We build approximate transmission conditions in order to replace the layer by these conditions on the boundary of the interior material. We deal with a weakly oscillating thin layer: the period of the oscillations is greater than the square root of the thinness. Our approach is essentially geometric and based on a suitable change of variable in the layer. This paper extends previous works [14], [15] of the former author, in which the layer had constant thickness.
\end{abstract}

\section{INTRODUCTION}

1.1. Motivations. The electromagnetic modelization of biological cells has become extremely important since several years, in particular in the biomedical research area. In the simple models of Fear, Stuchly or Foster and Schwan [10], [11], the biological cell is composed of a conducting cytoplasm surrounded by a thin insulating membrane. When exposed to an electric field, a potential difference is induced across the cell membrane. This transmembrane potential (TMP) may be of sufficient magnitude to be biologically significant. In particular, if it overcomes a threshold value, complex phenomenons as electropermeabilization or electroporation may occur [19], [20]: some exterior molecules might be internalized inside the cell. These process hold great promises in oncology and gene therapy, particularly, to deliver drug molecules in cancer treatment.

This is the reason why several papers in the bioelectromagnetic research area deal with numerical modelizations of the cell (see for instance [13], [18],[17]) and with numerical computations of the TMP. Actually the main difficulties in the calculation of the TMP lie in the thinness of the membrane and in the high contrast between the electromagnetic parameters of the cytoplasm and the membrane.

In previous papers [16], [15], we proposed an asymptotic analysis to compute electromagnetic fields and particularly electric potentials in domains with thin layer of constant thickness. However, in certain circumstances, electric field imposed to the cell may destructure the membrane. This leads to a thin membrane with non constant thickness, and the calculation of the TMP is then quiet more difficult. This is the reason why we present here an asymptotic analysis of the electro-quasistatic potentials in time-harmonic regime ${ }^{1}$ in a biological cell with membrane of non constant thickness; we even allow that weak oscillations in the tangential variable may occur.

\footnotetext{
${ }^{1}$ Roughly speaking, the electro-quasistatic equation in time-harmonic regime is the steady state voltage with complex parameters intead of real coefficients.
} 
Since we are definitely interested in the calculation of the transmembrane potential, the asymptotic expansions of the steady state voltage potentials far from the thin layer, which have been developped by Ammari, Vogelius et al. (see for instantce [3], [4], [5] or [7]) may not be used. Achdou et al. [2], Valentin et al. [12] or Abboud and Ammari [1] presented in previous papers how to build approximate boundary conditions for a highly oscillating thin membrane ${ }^{2}$, when a homogeneous Dirichlet boundary condition is imposed on the rough boundary. Here we are interested in approximate transmission conditions since our cell in embedded in an ambient, moreover, we deal with a weakly oscillating thin membrane. Once again previous analysis may not be applied.

Our asymptotic analysis is closed to those perfomed in [16]. Roughly speaking, it is based on a suitable change of variable in the membrane in order to write the explicit dependence of the studied differential operator in terms of the small parameter (the thinness of the membrane).

Throughout this paper, we consider a material composed of an interior domain surrounded by a thin membrane. This material, representing a biological cell, is embedded in an ambient medium. A voltage potential is imposed to the boundary of the ambient medium and we study the asymptotic behavior of the electroquasistatic voltage potential in the three domains (ambient medium, thin layer and cytoplasm) for the thinness of the membrane tending to zero. We build appropriate transmission conditions on the boundary of the cytoplasm in order to remove the thin layer from the problem. Actually, the influency of the layer is approached by these transmission conditions. To justify our asymptotic expansion, we estimate the $L^{2}$-error between the exact solution and the approximate solution.

This paper is structured as follows. In Section 2, we present the geometry of the problem and we perform our suitable change of variables. Then, in Section 3 we derive formally our asymptotics. This asymptotic expansion is then proved with error estimates, in Section 5.1.

1.2. The studied problem. Let $\Omega$ be a smooth bounded bidimensional domain (see Fig. 1), composed of a smooth interior domain $\mathcal{O}^{i}$ surrounded by a thin membrane $\mathcal{O}_{\delta}$ and of a ambient medium $\mathcal{O}_{\delta}^{e}$, where the domain $\overline{\mathcal{O}^{i} \cup \mathcal{O}_{\delta}}$ is embedded;

$$
\Omega=\mathcal{O}^{i} \cup \mathcal{O}_{\delta} \cup \mathcal{O}_{\delta}^{e} .
$$

We do not suppose that the thickness of the membrane is constant, we even allow that some parts of the membrane may be weakly oscillating.

Denote by $\Gamma$ the boundary of $\mathcal{O}^{i}$, which is supposed to be smooth and by $\Gamma_{\delta}^{r}$ the weakly oscillating (rough) boundary of $\mathcal{O}_{\delta}$. We suppose that the period of the oscillations is greater than the square root of the thin layer: this is the reason why the thin layer is said to be weakly oscillating.

Let $\sigma_{m}, \sigma_{i}$ and $\sigma_{e}$ be three non null complex parameters with positive real part. Denote by $\sigma_{\delta}$ the following piecewise constant functions

$$
\forall x \in \Omega, \quad \sigma_{\delta}(x)=\left\{\begin{array}{l}
\sigma_{i}, \text { if } x \in \mathcal{O}^{i} \\
\sigma_{m}, \text { if } x \in \mathcal{O}_{\delta}, \\
\sigma_{e}, \text { if } x \in \mathcal{O}_{\delta}^{e}
\end{array}\right.
$$

\footnotetext{
${ }^{2}$ These authors dealt with periods of the oscillations of the same length as the thickness of the membrane
} 


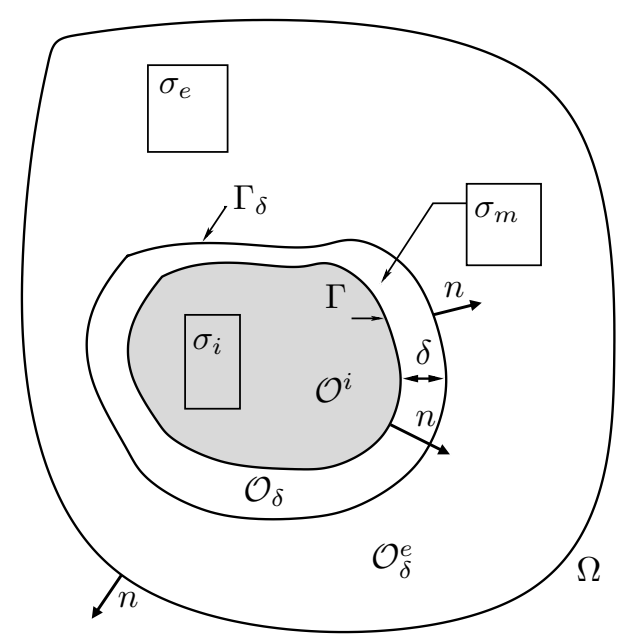

FIGURE 1. Geometric and dielectric data.

We would like to understand the behavior for $\delta$ tending to zero of the solution $V_{\delta}$ to the following problem :

$$
\begin{aligned}
\nabla \cdot\left(\sigma_{\delta} \nabla V_{\delta}\right) & =0 \text { in } \Omega, \\
\left.V_{\delta}\right|_{\partial \Omega} & =\varphi \text { on } \partial \Omega .
\end{aligned}
$$

The above function $V_{\delta}$ is well-defined and belongs to $H^{1+s}(\Omega)$ as soon as $\varphi$ belongs to $H^{1 / 2+s}(\partial \Omega), s \geq 0$. Observe that $V_{\delta}$ is continuous and its normal derivatives satisfy the following transmission conditions:

$$
\begin{gathered}
\left.\sigma_{m} \partial_{n} V_{\delta}\right|_{\Gamma^{+}}=\left.\sigma_{i} \partial_{n} V_{\delta}\right|_{\Gamma^{-}}, \\
\left.\sigma_{m} \partial_{n} V_{\delta}\right|_{\Gamma_{\delta}^{r-}}=\left.\sigma_{e} \partial_{n} V_{\delta}\right|_{\Gamma_{\delta}^{r+}} .
\end{gathered}
$$

We obtain in this paper, the approximate transmission conditions on $\Gamma$, which approaches the weakly oscillating thin layer, when the thickness of the membrane tends to zero.

1.3. Main result. The orientation of the boundary $\Gamma$ of $\mathcal{O}^{i}$ is the trigonometric orientation. To simplify, we suppose that the length of $\Gamma$ equals 1 . We denote by $\mathbb{T}$ the flat torus:

$$
\mathbb{T}=\mathbb{R} / \mathbb{Z}
$$

Since $\Gamma$ is smooth, we can parameterize it by a function $\Psi$ of class $\mathscr{C}^{\infty}$ from $\mathbb{T}$ to $\mathbb{R}^{2}$ satisfying:

$$
\forall \theta \in \mathbb{T}, \quad\left|\Psi^{\prime}(\theta)\right|=1,
$$

and therefore

$$
\Gamma=\{\Psi(\theta), \theta \in \mathbb{T}\} .
$$

Let $f$ be a quasiperiodic smooth function defined on $\mathbb{T} \times \mathbb{R}$ such that:

$$
\begin{aligned}
& \forall(x, y) \in \mathbb{T} \times \mathbb{R}, \quad f(x, y+1)=f(x, y), \\
& \forall(x, y) \in \mathbb{T} \times \mathbb{R}, \quad|f(x, y)| \leq M, \text { for a given constant } M>0 .
\end{aligned}
$$


We suppose there exists $\alpha \in[0,1 / 2)$ such that the thin membrane is parameterized as follows:

$$
\mathcal{O}_{\delta}=\left\{\Psi(\theta)+\delta \eta f\left(\theta, \frac{\theta}{\delta^{\alpha}}\right) n(\theta), \quad(\eta, \theta) \in[0,1] \times \mathbb{T}\right\} .
$$

Here $n(\theta)$ is the unitary exterior normal to $\Gamma$ at the point $\Psi(\theta)$. If $\alpha>0$, we denote by

$$
\forall x \in \Gamma, \quad \bar{g}(x)=\left(\int_{0}^{1} f(., y) \mathrm{d} y\right) \circ \Psi^{-1}(x),
$$

while for $\alpha=0, \bar{g}$ denotes

$$
\forall x \in \Gamma, \quad \bar{g}=f \circ \Psi^{-1}(x) .
$$

Suppose that $\varphi$ belongs to $H^{7 / 2}(\partial \Omega)$, and let $\left(v_{0}^{e}, v_{0}^{i}\right)$ satisfy:

$$
\left\{\begin{array}{l}
\Delta v_{0}^{e}=0, \text { in } \mathcal{O}^{e}, \\
\Delta v^{i}=0, \text { in } \mathcal{O}^{i},
\end{array}\right.
$$

with transmission conditions

$$
\begin{aligned}
& \left.v_{0}^{i}\right|_{\Gamma^{-}}=\left.v_{0}^{e}\right|_{\Gamma^{+}}, \\
& \left.\sigma_{i} \partial_{n} v_{0}^{i}\right|_{\Gamma^{-}}=\left.\sigma_{e} \partial_{n} v_{0}^{e}\right|_{\Gamma^{+}},
\end{aligned}
$$

and with the boundary condition

$$
\left.v_{0}^{e}\right|_{\partial \Omega}=\varphi,
$$

and define

$$
\bar{v}_{1}=\left\{\begin{array}{l}
\bar{v}_{1}^{e}, \text { in } \Omega \backslash \mathcal{O}^{i}, \\
\bar{v}_{1}^{i}, \text { in } \mathcal{O}^{i}
\end{array}\right.
$$

where:

$$
\left\{\begin{array}{l}
\Delta \bar{v}_{1}^{e}=0, \text { in } \Omega \backslash \mathcal{O}^{i}, \\
\Delta \bar{v}_{1}^{i}=0, \text { in } \mathcal{O}^{i}, \\
\left.\bar{v}_{1}^{e}\right|_{\partial \Omega}=0,
\end{array}\right.
$$

with the transmission conditions

$$
\begin{aligned}
& \left.\sigma_{i} \partial_{n} \bar{v}_{1}^{i}\right|_{\Gamma^{-}}-\left.\sigma_{e} \partial_{n} \bar{v}_{1}^{e}\right|_{\Gamma^{+}}=-\left(\sigma_{e}-\sigma_{m}\right) \partial_{t}\left(\left.\bar{g} \partial_{t} v_{0}^{i}\right|_{\Gamma^{-}}\right), \\
& \left.\bar{v}_{1}^{i}\right|_{\Gamma^{-}}-\left.\bar{v}_{1}^{e}\right|_{\Gamma^{+}}=\left.\bar{g}\left(\frac{1}{\sigma_{e}}-\frac{1}{\sigma_{m}}\right) \sigma_{i} \partial_{n} v_{0}^{i}\right|_{\Gamma^{-}} .
\end{aligned}
$$

We have the following theorem.

Theorem 1.1 (Main result). Suppose that $\alpha \in(0,1 / 2)$. The above functions $\left(v_{0}^{e}, v_{0}^{i}\right)$ and $\left(v_{1}^{e}, v_{1}^{i}\right)$ belong respectively to

$$
H^{4}\left(\mathcal{O}^{e}\right) \times H^{4}\left(\mathcal{O}^{i}\right), \quad \text { and to } \quad H^{3}\left(\mathcal{O}^{e}\right) \times H^{3}\left(\mathcal{O}^{i}\right) .
$$

Denote by $\overline{V_{a p p}}$ the following function:

$$
\bar{V}_{\text {app }}= \begin{cases}v_{0}^{i}+\delta \bar{v}_{1}^{i}, & \text { in } \mathcal{O}^{i}, \\ v_{0}^{e}+\delta \bar{v}_{1}^{e}, & \text { in } \Omega \backslash \mathcal{O}^{i} .\end{cases}
$$


Then we have the following estimates:

$$
\left\|V_{\delta}-\bar{V}_{a p p}\right\|_{L^{2}\left(\mathcal{O}^{i}\right)}=O\left(\delta^{1+\alpha / 2}+\delta^{3 / 2-\alpha}\right),
$$

and for all bounded domain $\omega$ such that $\bar{\omega} \subset \mathcal{O}_{\delta}^{e}$,

$$
\left\|V_{\delta}-\bar{V}_{a p p}\right\|_{L^{2}(\omega)}=O\left(\delta^{1+\alpha / 2}+\delta^{3 / 2-\alpha}\right) .
$$

Without any oscillation on $\Gamma_{\delta}^{r}$, i.e. if $\alpha=0$, we have the following estimates, which are more precise.

Theorem 1.2 (Without oscillations). Suppose that $\Gamma_{\delta}^{r}$ has no oscillating parts, then the following estimates hold:

$$
\left\|V_{\delta}-\bar{V}_{a p p}\right\|_{L^{2}\left(\mathcal{O}^{i}\right)}=O\left(\delta^{3 / 2}\right),
$$

and for all bounded domain $\omega$ such that $\bar{\omega} \subset \mathcal{O}_{\delta}^{e}$,

$$
\left\|V_{\delta}-\bar{V}_{a p p}\right\|_{L^{2}(\omega)}=O\left(\delta^{3 / 2}\right) .
$$

Remark 1.3. According to the previous theorems, to approach the potential $V_{\delta}$ defined by (1)-(2) with an accuracy of order $o(\delta)$, we first need the calculation of the terms $\left(v_{0}^{e}, v_{0}^{i}\right)$ and then we obtain $\left(v_{1}^{e}, v_{1}^{i}\right)$. The approximate potential is then the sum $\left(v_{0}^{e}+\delta v_{1}^{e}, v_{0}^{i}+\delta v_{1}^{i}\right)$. In order to compute only one problem, it is classical to write approximated boundary conditions. We leave the reader showing that the potential $\overline{v_{a p p}}$ satisfying the following problem:

$$
\begin{aligned}
& \Delta \bar{v}_{a p p}=0, \text { in } \Omega \backslash \mathcal{O}^{i}, \\
& \Delta \bar{v}_{a p p}=0, \text { in } \mathcal{O}^{i}, \\
& \left.\bar{v}_{a p p}\right|_{\partial \Omega}=\varphi,
\end{aligned}
$$

with the following approximated boundary conditions deduced from (6)-(7):

$$
\begin{aligned}
& \left.\sigma_{i} \partial_{n} \bar{v}_{a p p}\right|_{\Gamma^{-}}-\left.\sigma_{e} \partial_{n} \bar{v}_{a p p}\right|_{\Gamma^{+}}=-\delta\left(\sigma_{e}-\sigma_{m}\right) \partial_{t}\left(\left.\bar{g} \partial_{t} v_{a p p}\right|_{\Gamma^{-}}\right), \\
& \left.\bar{v}_{a p p}\right|_{\Gamma^{-}}-\left.\bar{v}_{a p p}\right|_{\Gamma^{+}}=\left.\delta \bar{g}\left(\frac{1}{\sigma_{e}}-\frac{1}{\sigma_{m}}\right) \sigma_{i} \partial_{n} v_{a p p}\right|_{\Gamma^{-}},
\end{aligned}
$$

is unique and satisfies the same estimates as $\left(v_{0}^{e}+\delta v_{1}^{e}, v_{0}^{i}+\delta v_{1}^{i}\right)$ in each domain $\mathcal{O}_{\delta}^{e}$ and $\mathcal{O}^{i}$.

To prove the estimates, it suffices to develop with respect to $\delta$ the above potential $\overline{v_{a p p}}$. We easily see that the first two terms are exactly the above terms $\left(v_{0}^{e}, v_{0}^{i}\right)$ and $\left(v_{1}^{e}, v_{1}^{i}\right)$, while the rest is $o(\delta)$. Existence and uniqueness of $\overline{v_{a p p}}$ comes from classical arguments.

Remark 1.4. Observe that the transmission condition $(7 \mathrm{c})$ leads directly to an estimate of the TMP across the membrane, with an accuracy of order $o(\delta)$. Therefore, to obtain the transmembrane potential at the order 1 , we only need to compute the electro-quasistatic potential without the thin membrane.

In this paper, we deal with a weakly oscillating membrane. However, in some modelization problems, it is interesting to deal with thin oscillating membrane, where the period of the oscillations as thin as the thickness of the membrane. The asymptotic expansion developped in this paper does not seem to be applicable, however, using a variationnal analysis, we may find the limit problem satisfied by the difference between the exact solution and the potential without membrane. These works are in progress and will be presented in a next paper [8]. 


\section{GeOmetry}

Denote by $\Phi$ the following map:

$$
\forall(\eta, \theta)[0,1] \times \mathbb{T}, \quad \Phi(\eta, \theta)=\Psi(\theta)+\delta f\left(\theta, \frac{\theta}{\delta^{\alpha}}\right) \eta n(\theta),
$$

where $\Psi$ is defined by (5) and let $\kappa$ be the curvature of $\Gamma$ in the curvilinear coordinate. Let $\delta_{0}$ belong to $(0,1)$ such that:

$$
\delta_{0}<\frac{1}{\|\kappa f\|_{\infty}} .
$$

Thus for all $\delta$ in $\left[0, \delta_{0}\right]$, there exists an open intervall $I$ containing $(0,1)$ such that $\Phi$ is a smooth diffeomorphism from $I \times \mathbb{R} / \mathbb{Z}$ to its image, which is a neighborhood of the membrane. Let $\left(g_{i j}\right)_{i, j=1}^{2}$ be the matrix describing the Euclidean metric in $(\eta, \theta)$-coordinates

$$
g_{11} \mathrm{~d} \eta^{2}+g_{22} \mathrm{~d} \theta^{2}+2 g_{12} \mathrm{~d} \eta \mathrm{d} \theta .
$$

The coefficients $\left(g_{i j}\right)_{i, j=1,2}$ are defined by (see for example Dubrovin et al. [9]:

$$
g_{11}=<\partial_{\eta} \Phi, \partial_{\eta} \Phi>, g_{22}=<\partial_{\eta} \Phi, \partial_{\eta} \Phi>, g_{12}=g_{21}=<\partial_{\eta} \Phi, \partial_{\theta} \Phi>
$$

where $<., .>$ denotes the Euclidean scalar product of $\mathbb{R}^{2}$.

Denote by $\nabla_{\alpha} \cdot f$ and $\nabla_{\alpha}^{2} \cdot f$ the following quantities:

$$
\begin{aligned}
& \nabla_{\alpha} \cdot f\left(\theta, \theta / \delta^{\alpha}\right)=\left.\partial_{x} f\right|_{\left(\theta, \theta / \delta^{\alpha}\right)}+\left.\frac{1}{\delta^{\alpha}} \partial_{y} f\right|_{\left(\theta, \theta / \delta^{\alpha}\right)} \\
& \nabla_{\alpha}^{2} \cdot f\left(\theta, \theta / \delta^{\alpha}\right)=\left.\partial_{x}^{2} f\right|_{\left(\theta, \theta / \delta^{\alpha}\right)}+\left.\frac{2}{\delta^{\alpha}} \partial_{x} \partial_{y} f\right|_{\left(\theta, \theta / \delta^{\alpha}\right)}+\left.\frac{1}{\delta^{2 \alpha}} \partial_{y}^{2} f\right|_{\left(\theta, \theta / \delta^{\alpha}\right)} .
\end{aligned}
$$

We have

$$
\begin{aligned}
& g_{11}=\left(\delta f\left(\theta, \theta / \delta^{\alpha}\right)\right)^{2} \\
& g_{22}=\left(1+\delta f\left(\theta, \theta / \delta^{\alpha}\right) \eta \kappa(\theta)\right)^{2}+\left(\eta \delta \nabla_{\alpha} \cdot f\left(\theta, \theta / \delta^{\alpha}\right)\right)^{2}, \\
& g_{12}=g_{21}=\eta \delta^{2} f\left(\theta, \theta / \delta^{\alpha}\right) \nabla_{\alpha} \cdot f\left(\theta, \theta / \delta^{\alpha}\right)
\end{aligned}
$$

and let $|G|$ be:

$$
|G|=g_{11} g_{22}-g_{12}^{2}=\delta^{2} f^{2}\left(\theta, \theta / \delta^{\alpha}\right)\left(1+\eta \kappa(\theta) \delta f\left(\theta, \theta / \delta^{\alpha}\right)\right)^{2} .
$$

Write now Laplacian operator in $(\eta, \theta)$-coordinates. Using the formulae of differential geometry ${ }^{3}$ (see for instance Appendix of [16]), we have:

$$
\Delta_{\eta, \theta}=\frac{1}{\sqrt{|G|}}\left\{\partial_{\eta}\left(\frac{1}{\sqrt{|G|}}\left(g_{22} \partial_{\eta}-g_{12} \partial_{\theta}\right)\right)+\partial_{\theta}\left(\frac{1}{\sqrt{|G|}}\left(-g_{12} \partial_{\eta}+g_{11} \partial_{\theta}\right)\right)\right\} .
$$

\footnotetext{
${ }^{3}$ Without any knowledge in diffential geometry area but simply using the change of variables $(x, y) \rightarrow(\eta, \theta)$, the above expression of the Laplacian might also be derived by tedious calculations.
} 
Therefore we have

$$
\begin{aligned}
|G| \Delta_{\eta, \theta} & =g_{22} \partial_{\eta}^{2}+g_{11} \partial_{\theta}^{2}-2 g_{12} \partial_{\eta} \partial_{\theta} \\
& +\left(\partial_{\eta} g_{22}-\partial_{\theta} g_{12}+g_{12} \frac{\partial_{\theta}|G|}{2|G|}-g_{22} \frac{\partial_{\eta}|G|}{2|G|}\right) \partial_{\eta} \\
& +\left(\partial_{\theta} g_{11}-\partial_{\eta} g_{12}+g_{12} \frac{\partial_{\eta}|G|}{2|G|}-g_{11} \frac{\partial_{\theta}|G|}{2|G|}\right) \partial_{\theta}
\end{aligned}
$$

On the boundary $\Gamma$, the normal derivative equals:

$$
\left.\left.\partial_{n}\right|_{\Gamma} \circ \Phi\right|_{\eta=0}=\left.\frac{1}{\delta f\left(\theta, \theta / \delta^{\alpha}\right)} \partial_{\eta}\right|_{\eta=0},
$$

and on the oscillating boundary $\Gamma_{\delta}^{r}$,

$$
\left.\left.\partial_{n}\right|_{\Gamma_{\delta}^{r}} \mathrm{o} \Phi\right|_{\eta=1}=\left.\frac{1}{\sqrt{|G|}}\left\{\sqrt{g_{22}} \partial_{\eta}-\frac{g_{12}}{\sqrt{g_{22}}} \partial_{\theta}\right\}\right|_{\eta=1} .
$$

Remark 2.1. In this paper we suppose that $\alpha$ belongs to $[0,1 / 2)$. Observe that if $\alpha$ belongs to $[1 / 2,1)$, the multiplication by the above function $\nabla_{\alpha}^{2} \cdot f$ (or equivalently the multiplication by $\left.\left(\nabla_{\alpha} \cdot f\right)^{2}\right)$ is of order -1 with respect to the power of $\delta$. This case will be consider later on.

\section{Formal Asymptotics}

This section is devoted to derive formally the asymptotic expansion of $V$.

Let us set our ansatz:

$$
\begin{aligned}
v^{e} & =v_{0}^{e}+\delta v_{1}^{e}+\cdots, \\
v^{c} & =v_{0}^{i}+\delta v_{1}^{i}+\cdots \\
v^{m} & =v_{0}^{m}+\delta v_{1}^{m}+\delta^{2} v_{2}^{m}+\cdots
\end{aligned}
$$

A priori, each functions in the above equalities depends on $\theta / \delta^{\alpha}$, but we expect that this dependency will be explicit. For all $k \geq 0$, we set:

$$
\begin{aligned}
& \Delta v_{k}^{e}=0, \text { in } \mathcal{O}^{e}, \\
& \Delta v_{k}^{i}=0, \text { in } \mathcal{O}^{i}, \\
& \left.v_{k}^{e}\right|_{\partial \Omega}=\delta_{0, k} \varphi, \text { on } \partial \Omega .
\end{aligned}
$$

We order the terms of the same power of $\delta$ in the expressions of $\Delta_{\eta, \theta}$ and of the normal derivatives. We emphasize that for all $k \geq 0, v_{k}^{m}$ depends a priori on $\eta$, $\theta / \delta^{\alpha}$ and $\theta$. Using the following equalities:

$$
\begin{aligned}
\frac{\partial_{\eta}|G|}{2|G|} & =\frac{\delta \kappa(\theta) f\left(\theta, \theta / \delta^{\alpha}\right)}{1+\eta \kappa(\theta) \delta f\left(\theta, \theta / \delta^{\alpha}\right)}, \\
\frac{\partial_{\theta}|G|}{2|G|} & =\frac{\nabla_{\alpha} \cdot f\left(\theta, \theta / \delta^{\alpha}\right)}{f\left(\theta, \theta / \delta^{\alpha}\right)}+\eta \delta \frac{\kappa(\theta) \nabla_{\alpha} \cdot f\left(\theta, \theta / \delta^{\alpha}\right)+\kappa^{\prime}(\theta) f\left(\theta, \theta / \delta^{\alpha}\right)}{1+\eta \kappa(\theta) \delta f\left(\theta, \theta / \delta^{\alpha}\right)}
\end{aligned}
$$


we infer, with the notation $\tau=\left(\theta, \theta / \delta^{\alpha}\right)$,

$$
\begin{aligned}
|G| \Delta_{\eta, \theta} & =\partial_{\eta}^{2}+\delta \kappa(\theta) f(\tau)\left(2 \eta \partial_{\eta}^{2}+\partial_{\eta}\right) \\
& +\delta^{2}\left\{\left(\eta^{2}\left(\nabla_{\alpha} \cdot f(\tau)\right)^{2}+(\eta \kappa(\theta) f(\tau))^{2}\right) \partial_{\eta}^{2}+f^{2}(\tau) \partial_{\theta}^{2}\right. \\
& -2 \eta f(\tau) \nabla_{\alpha} \cdot f(\tau) \partial_{\theta} \partial_{\eta}+\eta\left(\kappa^{2}(\theta) f^{2}(\tau)+2\left(\nabla_{\alpha} \cdot f(\tau)\right)^{2}\right. \\
& \left.\left.-f(\tau) \nabla_{\alpha}^{2} \cdot f(\tau)\right) \partial_{\eta}\right\} \\
& +\delta^{3} R(\eta, \tau),
\end{aligned}
$$

where $R$ is an operator of order 1 on $[0,1] \times \mathbb{T}$ defined by:

(19) $R(\eta, \tau)=\frac{\eta f(\tau)}{1+\kappa(\theta) \eta \delta f(\tau)}\left\{\eta\left(\kappa^{\prime}(\theta) \nabla_{\alpha} f-f^{2}(\tau) \kappa^{3}(\theta)\right) \partial_{\eta}-\kappa^{\prime}(\theta) f^{2}(\tau) \partial_{\theta}\right\}$.

We also have:

$$
\begin{aligned}
\left.\left.\partial_{n}\right|_{\Gamma_{\delta}^{r}} \mathrm{O} \Phi\right|_{\eta=1} & =\frac{1}{\delta f(\tau)}\left\{\partial_{\eta}\right. \\
& \left.+\delta^{2} \nabla_{\alpha} \cdot f(\tau)\left(\frac{\nabla_{\alpha} \cdot f(\tau)}{2} \partial_{\eta}-f(\tau) \partial_{\theta}\right)+T(\tau)\right\}\left.\right|_{\eta=1},
\end{aligned}
$$

where $T$ is the following differential operator defined on $\mathbb{T}$ by:

$$
\begin{aligned}
T(\tau) & =\left(\sqrt{1+\delta^{2}\left(\nabla_{\alpha} \cdot f(\tau)\right)^{2} /(1+\kappa \delta f(\tau))^{2}}-1-\delta^{2}\left(\nabla_{\alpha} \cdot f(\tau)\right)^{2} / 2\right) \partial_{\eta} \\
& -\delta^{2} f(\tau) \nabla_{\alpha} \cdot f(\tau)\left\{\frac{1}{(1+\kappa \delta f(\tau))^{2} \sqrt{1+\left(\frac{\delta \nabla_{\alpha} \cdot f(\tau)}{1+\kappa \delta f(\tau)}\right)^{2}}}-1\right\} \partial_{\theta} .
\end{aligned}
$$

To obtain the approximated transmission conditions on the smooth boundary $\Gamma$, we need to extend formally $v^{e}$ in the membrane. Using a Taylor expansion in the variable $\eta$, we obtain,

$$
\left.v^{e} \mathrm{o} \Phi\right|_{\eta=1}=\left.v_{0}^{e} \mathrm{o} \Phi\right|_{\eta=0}+\delta\left(\left.v_{1}^{e} \mathrm{o} \Phi\right|_{\eta=0}+\left.f(\tau) \partial_{n} v_{0}^{e} \mathrm{o} \Phi\right|_{\eta=0}\right)+\cdots,
$$

and similarly, denoting by $\partial_{t}$ the tangential derivative on $\Gamma$ we have

$$
\begin{aligned}
\left.\partial_{n} v^{e} \mathrm{o} \Phi\right|_{\eta=1} & =\left.\partial_{n} v_{0}^{e} \mathrm{O} \Phi\right|_{\eta=0}+\delta\left(\left.\partial_{n} v_{1}^{e} \mathrm{O} \Phi\right|_{\eta=0}\right. \\
& \left.-\nabla_{\alpha} .\left.f(\tau) \partial_{t} v_{0}^{e} \mathrm{O} \Phi\right|_{\eta=0}+\left.f(\tau) \partial_{n}^{2} v_{0}^{e} \mathrm{o} \Phi\right|_{\eta=0}\right)+\cdots
\end{aligned}
$$


To identify the first two terms of the asymptotic expansion of the steady state potentials, we need few notations. Let $\mathfrak{K}$ be the curvature of $\Gamma$ in Euclidean coordinates:

$$
\forall x \in \Gamma, \quad \mathfrak{K}(x)=\kappa \circ \Psi^{-1}(x),
$$

and define $g_{\delta, \alpha}$ on $\Gamma$ as follows:

$$
\forall \theta \in \mathbb{T}, \quad g_{\delta, \alpha} \mathrm{O} \Psi(\theta)=f\left(\theta, \frac{\theta}{\delta^{\alpha}}\right),
$$

therefore, for all $\theta \in \mathbb{T}$,

$$
\partial_{t} g_{\delta, \alpha} \circ \Psi(\theta)=\nabla_{\alpha} . f\left(\theta, \frac{\theta}{\delta^{\alpha}}\right) .
$$

Now, we just have to identify the term with the same power of $\delta$.

- $\underline{0}^{\text {th }}$ order term. We have necessarily:

$$
\partial_{\eta}^{2} v_{0}^{m}=0,
$$

and $\left.\partial_{\eta} v_{0}^{m}\right|_{\eta=0}=0$, hence $v_{0}^{m}=v_{0}^{m}\left(\theta, \theta / \delta^{\alpha}\right)$ and therefore, $\left.v_{0}^{e}\right|_{\Gamma^{+}}=\left.v_{0}^{i}\right|_{\Gamma^{-}}$.

- First order term. Using $\partial_{\eta} v_{0}^{m} \equiv 0$, we obtain:

$$
\partial_{\eta}^{2} v_{1}^{m}=0
$$

hence $\partial_{\eta} v_{1}^{m}$ is constant with respect to $\eta$. According to transmission conditions (3), by identification, we necessarily have:

$$
\left.\sigma_{m} \partial_{\eta} v_{1}^{m}\right|_{\eta=0}=\left.f(\tau) \sigma_{i} \partial_{n} v_{0}^{i}\right|_{\Gamma^{-}} \mathrm{o} \Psi,
$$

and

$$
\left.\sigma_{m} \partial_{\eta} v_{1}^{m}\right|_{\eta=1}=\left.f(\tau) \sigma_{e} \partial_{n} v_{0}^{e}\right|_{\Gamma^{+}} \mathrm{o} \Psi
$$

we infer :

$$
\left.\sigma_{e} \partial_{n} v_{0}^{e}\right|_{\Gamma^{+}}=\left.\sigma_{i} \partial_{n} v_{0}^{i}\right|_{\Gamma^{-}}
$$

which determine uniquely $v_{0}^{e}$ and $v_{0}^{i}$. Moreover, we have $v_{0}^{m}=v_{0}^{i}$ o $\Psi$, thus $v_{0}^{m}$ depends only on $\theta$, and

$$
\partial_{\eta} v_{1}^{m}=\left.f\left(\theta, \theta / \delta^{\alpha}\right) \frac{\sigma_{i}}{\sigma_{m}} \partial_{n} v_{0}^{i}\right|_{\Gamma^{-}} \mathrm{o} \Psi .
$$

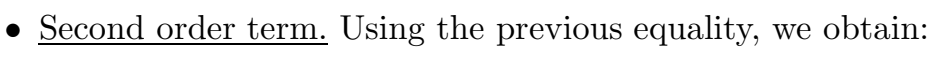

$$
\partial_{\eta}^{2} v_{2}^{m}+\kappa f \partial_{\eta} v_{1}^{m}+f^{2} \partial_{\theta}^{2} v_{0}^{m}=0,
$$

hence $\partial_{\eta} v_{2}^{m}$ equals:

$$
\begin{aligned}
\partial_{\eta} v_{2}^{m} & =-\eta f^{2}\left(\theta, \theta / \delta^{\alpha}\right)\left(\left.\kappa \frac{\sigma_{i}}{\sigma_{m}} \partial_{n} v_{0}^{i}\right|_{\Gamma^{-}} \mathrm{O} \Psi+\left.\partial_{t}^{2} v_{0}^{i}\right|_{\Gamma^{-}} \mathrm{O} \Psi\right) \\
& +\left.f\left(\theta, \theta / \delta^{\alpha}\right) \frac{\sigma_{i}}{\sigma_{m}} \partial_{n} v_{1}^{i}\right|_{\Gamma^{-}} \mathrm{o} \Psi .
\end{aligned}
$$

Since we have:

$$
\begin{aligned}
\frac{1}{f(\tau)}\left(\left.\partial_{\eta} v_{2}^{m}\right|_{\eta=1}-\left.f(\tau) \nabla_{\alpha} \cdot f(\tau) \partial_{t} v_{0}^{i}\right|_{\Gamma^{-}}\right) & =\frac{\sigma_{e}}{\sigma_{m}}\left(\left.\partial_{n} v_{1}^{e}\right|_{\Gamma^{+}}-\left.\nabla_{\alpha} \cdot f(\tau) \partial_{t} v_{0}^{e}\right|_{\Gamma^{+}}\right. \\
& \left.+\left.f(\tau) \partial_{n}^{2} v_{0}^{e}\right|_{\Gamma^{+}}\right)
\end{aligned}
$$


and since

$$
\left.\partial_{n}^{2} v_{0}^{e}\right|_{\Gamma^{+}}=-\left.\partial_{t}^{2} v_{0}^{e}\right|_{\Gamma^{+}}-\left.\mathfrak{K} \partial_{n} v_{0}^{e}\right|_{\Gamma^{+}},
$$

we infer easily the transmission conditions between $v_{1}^{e}$ and $v_{1}^{i}$ :

$$
\left.\sigma_{e} \partial_{n} v_{1}^{e}\right|_{\Gamma^{+}}-\left.\sigma_{i} \partial_{n} v_{1}^{i}\right|_{\Gamma^{-}}=\left(\sigma_{e}-\sigma_{m}\right) \partial_{t}\left(\left.g_{\delta, \alpha} \partial_{t} v_{0}^{i}\right|_{\Gamma^{-}}\right)
$$

and

$$
\left.v_{1}^{e}\right|_{\Gamma^{+}}-\left.v_{1}^{i}\right|_{\Gamma^{-}}=-\left.g_{\delta, \alpha}\left(\frac{1}{\sigma_{e}}-\frac{1}{\sigma_{m}}\right) \sigma_{i} \partial_{n} v_{0}^{i}\right|_{\Gamma^{-}} .
$$

Moreover, in the membrane, we have

$$
v_{1}^{m}=\left.\eta f\left(\theta / \delta^{\alpha}\right) \frac{\sigma_{i}}{\sigma_{m}} \partial_{n} v_{0}^{i}\right|_{\Gamma^{-}} \mathrm{o} \Psi+\left.v_{1}^{i}\right|_{\Gamma^{-}} \mathrm{o} \Psi .
$$

Therefore, formally, we have built the first two terms of the solution $v$ to problem (1)-(2). We summarize here our formal results.

- The $0^{\text {th }}$-order coefficients. The electric potentials $v_{0}^{e}$ and $v_{0}^{c}$ are defined by (6). In the membrane, the field $v_{0}^{m}$ equals:

$$
\forall(\eta, \theta) \in[0,1] \times \mathbb{T}, \quad v_{0}^{m}=\left.v_{0}^{i}\right|_{\Gamma^{-}} \mathrm{O} \Psi .
$$

- The first order coefficients. The potentials $v_{1}^{e}$ and $v_{1}^{i}$ are solution to the following problem in $\Omega$ :

$$
\left\{\begin{array}{l}
\Delta v_{1}^{e}=0, \text { in } \Omega \backslash \mathcal{O}^{i} \\
\Delta v_{1}^{i}=0, \text { in } \mathcal{O}^{i}, \\
\left.v_{1}^{e}\right|_{\partial \Omega}=0,
\end{array}\right.
$$

with the transmission conditions

$$
\begin{aligned}
\left.\sigma_{i} \partial_{n} v_{1}^{i}\right|_{\Gamma^{-}}-\left.\sigma_{e} \partial_{n} v_{1}^{e}\right|_{\Gamma^{+}} & =-\left(\sigma_{e}-\sigma_{m}\right) \partial_{t}\left(\left.g_{\delta, \alpha} \partial_{t} v_{0}^{i}\right|_{\Gamma^{-}}\right), \\
\left.v_{1}^{i}\right|_{\Gamma^{-}}-\left.v_{1}^{e}\right|_{\Gamma^{+}} & =\left.g_{\delta, \alpha}\left(\frac{1}{\sigma_{e}}-\frac{1}{\sigma_{m}}\right) \sigma_{i} \partial_{n} v_{0}^{i}\right|_{\Gamma^{-}} .
\end{aligned}
$$

In the membrane, we have:

$$
\forall(\eta, \theta) \in[0,1] \times \mathbb{T}, \quad v_{1}^{m}=\left.\eta f\left(\theta, \theta / \delta^{\alpha}\right) \frac{\sigma_{i}}{\sigma_{m}} \partial_{n} v_{0}^{i}\right|_{\Gamma^{-}} \mathrm{o} \Psi+\left.v_{1}^{i}\right|_{\Gamma^{-}} \mathrm{o} \Psi
$$

Observe that $v_{1}$ depends on $\delta / \alpha$, thus we have to find the limit problem of $v_{1}$. Remember the expression of $\partial_{\eta} v_{2}^{m}$ given in (20):

$$
\begin{aligned}
\partial_{\eta} v_{2}^{m} & =-\eta f^{2}\left(\theta, \theta / \delta^{\alpha}\right)\left(\left.\kappa \frac{\sigma_{i}}{\sigma_{m}} \partial_{n} v_{0}^{i}\right|_{\Gamma^{-}} \mathrm{o} \Psi+\left.\partial_{t}^{2} v_{0}^{i}\right|_{\Gamma^{-}} \mathrm{o} \Psi\right) \\
& +\left.f\left(\theta, \theta / \delta^{\alpha}\right) \frac{\sigma_{i}}{\sigma_{m}} \partial_{n} v_{1}^{i}\right|_{\Gamma^{-}} \mathrm{o} \Psi
\end{aligned}
$$

Let us give now useful estimates for $\left(v_{0}^{i}, v_{0}^{e}\right)$ and $\left(v_{1}^{i}, v_{1}^{e}\right)$. 
Lemma 3.1. Let $k_{0} \in \mathbb{N}^{*}$ and $\varphi$ belong to $H^{k_{0}-1 / 2}(\partial \Omega)$. Then we have the following regularity:

$$
\begin{aligned}
& \left(v_{0}^{e}, v_{0}^{i}\right) \in H^{k_{0}}\left(\Omega \backslash \mathcal{O}^{i}\right) \times H^{k}\left(\mathcal{O}^{i}\right), \\
& \left(v_{1}^{e}, v_{1}^{i}\right) \in H^{k_{0}-1}\left(\Omega \backslash \mathcal{O}^{i}\right) \times H^{3}\left(\mathcal{O}^{i}\right), \\
& v_{0}^{m} \in \mathcal{C}^{\infty}\left([0,1], H^{k_{0}-1 / 2}(\mathbb{T})\right), \\
& v_{1}^{m} \in \mathcal{C}^{\infty}\left([0,1], H^{k_{0}-3 / 2}(\mathbb{T})\right), \\
& \partial_{\eta} v_{2}^{m} \in \mathcal{C}^{\infty}\left([0,1], H^{k_{0}-3 / 2}(\mathbb{T})\right) .
\end{aligned}
$$

Moreover there exists a constant $C>0 \delta$-independent such that for $l=0, \cdots, k_{0}$,

$$
\begin{aligned}
& \left\|v_{0}^{i}\right\|_{H^{l}\left(\mathcal{O}^{i}\right)} \leq C|\varphi|_{H^{k_{0}-1 / 2}(\partial \Omega)}, \\
& \left\|v_{0}^{e}\right\|_{H^{l}\left(\Omega \backslash \mathcal{O}^{i}\right)} \leq C|\varphi|_{H^{k_{0}-1 / 2}(\partial \Omega)}, \\
& \sup _{\eta \in[0,1]}\left|v_{0}^{m}\right|_{H^{l-1 / 2}(\mathbb{T})} \leq C|\varphi|_{H^{k_{0}-1 / 2}(\partial \Omega)},
\end{aligned}
$$

and for $l=1, \cdots, k_{0}-1$,

$$
\begin{aligned}
& \left\|v_{1}^{i}\right\|_{H^{l}\left(\mathcal{O}^{i}\right)} \leq C \delta^{-l \alpha}|\varphi|_{H^{k_{0}-1 / 2}(\partial \Omega)}, \\
& \left\|v_{1}^{e}\right\|_{H^{l}\left(\Omega \backslash \mathcal{O}^{i}\right)} \leq C \delta^{-l \alpha}|\varphi|_{H^{k_{0}-1 / 2}(\partial \Omega)}, \\
& \sup _{\eta \in[0,1]}\left|v_{1}^{m}\right|_{H^{l-3 / 2}(\mathbb{T})} \leq C \delta^{-l \alpha}|\varphi|_{H^{k_{0}-1 / 2}(\partial \Omega)}, \\
& \sup _{\eta \in[0,1]}\left|\partial_{\eta} v_{2}^{m}\right|_{H^{l-3 / 2}(\mathbb{T})} \leq C \delta^{-l \alpha}|\varphi|_{H^{k_{0}-1 / 2}(\partial \Omega)} .
\end{aligned}
$$

Proof. The estimates involving the $0^{\text {th }}$-order terms are well-known, since these terms do not take the thin oscillating layer into account. The estimates on $v_{1}^{m}$ and $\partial_{\eta} v_{2}^{m}$ easily come from the estimates on $v_{1}^{e}$ and $v_{1}^{i}$. Therefore we just have to prove the results for $v_{1}^{e}$ and $v_{1}^{i}$.

Observe that $\left(v_{1}^{e}, v_{1}^{i}\right)$ are defined in a domain without oscillation. Denote by $F_{1}$ and $F_{2}$ the two following functions

$$
F_{1}=-\left(\sigma_{e}-\sigma_{m}\right) \partial_{t}\left(\left.g_{\delta, \alpha} \partial_{t} v_{0}^{i}\right|_{\Gamma^{-}}\right),
$$

and

$$
F_{2}=\left.g_{\delta, \alpha}\left(\frac{1}{\sigma_{e}}-\frac{1}{\sigma_{m}}\right) \sigma_{i} \partial_{n} v_{0}^{i}\right|_{\Gamma^{-}} .
$$

Obviously, we have the following estimates, for $l=0, \cdots, k_{0}-1$ :

$$
\left|F_{1}\right|_{H^{l-1 / 2}\left(\partial \mathcal{O}^{i}\right)} \leq C \delta^{-(l+1) \alpha}|\varphi|_{H^{k_{0}-1 / 2}(\partial \Omega)},
$$

and

$$
\left|F_{2}\right|_{H^{l+1 / 2}\left(\partial \mathcal{O}^{i}\right)} \leq C \delta^{-(l+1) \alpha}|\varphi|_{H^{k_{0}-1 / 2}(\partial \Omega)} .
$$

Since $\left(v_{1}^{e}, v_{1}^{i}\right)$ satisfies:

$$
\left\{\begin{array}{l}
\Delta v_{1}^{e}=0, \text { in } \Omega \backslash \mathcal{O}^{i} \\
\Delta v_{1}^{i}=0, \text { in } \mathcal{O}^{i} \\
\left.v_{1}^{e}\right|_{\partial \Omega}=0
\end{array}\right.
$$


with the transmission conditions

$$
\begin{aligned}
& \left.\sigma_{i} \partial_{n} v_{1}^{i}\right|_{\Gamma^{-}}-\left.\sigma_{e} \partial_{n} v_{1}^{e}\right|_{\Gamma^{+}}=F_{1}, \\
& \left.v_{1}^{i}\right|_{\Gamma^{-}}-\left.v_{1}^{e}\right|_{\Gamma^{+}}=F_{2},
\end{aligned}
$$

using a well-known extension of Lemma 0.1 of [12] (see also [2]), we infer easily the following estimates, for $l=1, \cdots, k_{0}-1$ :

$$
\begin{aligned}
& \left\|v_{1}^{i}\right\|_{H^{l}\left(\mathcal{O}^{i}\right)} \leq C \delta^{-l \alpha}|\varphi|_{H^{k_{0}-1 / 2}(\partial \Omega)}, \\
& \left\|v_{1}^{e}\right\|_{H^{l}\left(\Omega \backslash \mathcal{O}^{i}\right)} \leq C \delta^{-l \alpha}|\varphi|_{H^{k_{0}-1 / 2}(\partial \Omega)},
\end{aligned}
$$

hence the lemma.

\section{Limit PROBLEM OF $v_{1}$}

If $\alpha=0$, the functions $v_{1}$ and $\bar{v}_{1}$ are equal, since no oscillation occurs on $\Gamma_{\delta}^{r}$. For $\alpha \in(0,1 / 2)$, the following lemma ensures that the solution $\bar{v}_{1}$ to problem (7) is the $L^{2}$-limit of $v_{1}$, for $\delta$ tending to zero.

Lemma 4.1. Suppose $\alpha \in(0,1 / 2)$ and let $\varphi$ belong to $H^{k_{0}-1 / 2}(\partial \Omega), k_{0} \geq 2$. Denote by:

$$
v_{1}=\left(v_{1}^{e}, v_{1}^{i}\right), \text { and } \quad \bar{v}_{1}=\left(\bar{v}_{1}^{e}, \bar{v}_{1}^{i}\right)
$$

the respective solutions to problem (22) and (7). Then,

$$
\left\|v_{1}-\bar{v}_{1}\right\|_{L^{2}(\Omega)}=O\left(\delta^{\alpha / 2}\right), \quad \text { for } \delta \text { tending to zero. }
$$

Remark 4.2. To prove of this lemma, we need a precise bound of the function $g_{\delta, \alpha}-\bar{g}$ in $H^{-1}(\Gamma)$. Actually, there exists a $\delta$-independent positive constant $C$ such that

$$
\left\|g_{\delta, \alpha}-\bar{g}\right\|_{H^{-1}(\Gamma)} \leq C \delta^{\alpha}
$$

Since the change of variable $x=\Psi(\theta)$ does not involve the small parameter $\delta^{\alpha}$, we just have to prove the above estimate for the function

$$
\theta \in \mathbb{T} \longrightarrow f\left(\theta, \theta / \delta^{\alpha}\right)-\int_{0}^{1} f(\theta, y) \mathrm{d} y
$$

Moreover, by a density argument, we suppose that $f(x, y)=f_{1}(x) f_{2}(y)$, where $f_{1}$ and $f_{2}$ are 1-periodic functions. Therefore,

$$
f\left(\theta, \theta / \delta^{\alpha}\right)-\int_{0}^{1} f(\theta, y) \mathrm{d} y=f_{1}(\theta) g_{2}\left(\theta / \delta^{\alpha}\right),
$$

where $g_{2}(y)=f_{2}(y)-\int_{0}^{1} f_{2}(s) \mathrm{d} s$. The following function $\mathscr{P}_{f}$ defined for $\theta \in[0,1]$ :

$$
\mathscr{P}_{f}(\theta)=\int_{0}^{\theta} f_{1}(t) g_{2}\left(t / \delta^{\alpha}\right) \mathrm{d} t-\theta \int_{0}^{1} f_{1}(t) g_{2}\left(t / \delta^{\alpha}\right) \mathrm{d} t,
$$

and extended by periodicity to $\mathbb{T}$ is a periodic primitive of

$$
\theta \in \mathbb{T} \longrightarrow f\left(\theta, \theta / \delta^{\alpha}\right)-\int_{0}^{1} f(\theta, y) \mathrm{d} y
$$

and a simple calculation shows that

$$
\left\|\mathscr{P}_{f}\right\|_{L^{2}(\mathbb{T})} \leq C \delta^{\alpha}
$$

from which we infer (24). 
We are now ready to proof the above lemma.

Proof of Lemma 4.1. Denote by $\tilde{\sigma}$, the function:

$$
\tilde{\sigma}= \begin{cases}\sigma_{i}, & \text { in } \mathcal{O}^{i}, \\ \sigma_{e}, & \text { in } \Omega \backslash \mathcal{O}^{i} .\end{cases}
$$

For all $\psi \in L^{2}(\Omega)$, denote by $\phi$ the solution in $H_{0}^{1}(\Omega)$ to the following problem:

$$
\nabla \cdot(\tilde{\sigma} \nabla \phi)=\psi, \quad \text { in } \Omega .
$$

Let $w_{1}$ equal $w_{1}=v_{1}-\bar{v}_{1}$. This function satisfies:

$$
\left\{\begin{array}{l}
\Delta w_{1}=0, \text { in } \Omega \backslash \mathcal{O}^{i}, \\
\Delta w_{1}=0, \text { in } \mathcal{O}^{i}, \\
\left.w_{1}\right|_{\partial \Omega}=0,
\end{array}\right.
$$

with the transmission conditions

$$
\begin{aligned}
& \left.\sigma_{i} \partial_{n} w_{1}\right|_{\Gamma^{-}}-\left.\sigma_{e} \partial_{n} w_{1}\right|_{\Gamma^{+}}=-\left(\sigma_{e}-\sigma_{m}\right) \partial_{t}\left(\left.\left(g_{\delta, \alpha}-\bar{g}\right) \partial_{t} v_{0}^{i}\right|_{\Gamma^{-}}\right), \\
& \left.w_{1}\right|_{\Gamma^{-}}-\left.v_{1}\right|_{\Gamma^{+}}=\left.\left(g_{\delta, \alpha}-\bar{g}\right)\left(\frac{1}{\sigma_{e}}-\frac{1}{\sigma_{m}}\right) \sigma_{i} \partial_{n} v_{0}^{i}\right|_{\Gamma^{-}} .
\end{aligned}
$$

Multiplication of (25) by $\phi$, and integration by parts now gives:

$$
\begin{aligned}
\int_{\Omega} w_{1}(x) \psi(x) \mathrm{d} x & =\int_{\Gamma}\left(g_{\delta, \alpha}(s)-\bar{g}(s)\right)\left(\left.\left.\left(\sigma_{e}-\sigma_{m}\right) \partial_{t} v_{0}^{i}\right|_{\Gamma^{-}}(s) \partial_{t} \phi\right|_{\Gamma^{-}}(s)\right. \\
& \left.+\left.\left.\left(\frac{1}{\sigma_{e}}-\frac{1}{\sigma_{m}}\right) \sigma_{i}^{2} \partial_{n} v_{0}^{i}\right|_{\Gamma^{-}}(s) \partial_{n} \phi\right|_{\Gamma^{-}}(s)\right) \mathrm{d} s
\end{aligned}
$$

Observe that the following bounds hold, for a $\delta$-independent positive constant $C$ :

$$
\begin{aligned}
& \left\|g_{\delta, \alpha}-\bar{g}\right\|_{L^{2}(\Gamma)} \leq C, \\
& \left\|g_{\delta, \alpha}-\bar{g}\right\|_{H^{-1}(\Gamma)} \leq C \delta^{\alpha},
\end{aligned}
$$

according to (24). Using the well-known interpolation theorem, it follows

$$
\left\|g_{\delta, \alpha}-\bar{g}\right\|_{H^{-1 / 2}(\Gamma)} \leq C \delta^{\alpha / 2} .
$$

From (26), there exists a $\delta$-independent constant $C$ such that :

$$
\begin{aligned}
\left|\int_{\Omega} w_{1}(x) \psi(x) \mathrm{d} x\right| & \leq C \delta^{\alpha / 2}\|\phi\|_{H^{2}(\Omega)} \\
& \leq C \delta^{\alpha / 2}\|\psi\|_{L^{2}(\Omega)}
\end{aligned}
$$

hence the lemma.

\section{ERror estimates}

The following theorem justify our asymptotic expansion. 
Theorem 5.1. We suppose that the boundary data $\varphi$ belongs to $H^{7 / 2}(\partial \Omega)$. Denote by $V_{\text {app }}$ the following function:

$$
V_{a p p}=\left\{\begin{array}{l}
v_{0}^{e}+\delta v_{1}^{e}, \text { in } \mathcal{O}_{\delta}^{e} \\
v_{0}^{i}+\delta v_{1}^{i}, \text { in } \mathcal{O}^{i} \\
\left(v_{0}^{m}+\delta v_{1}^{m}+\delta^{2} \int_{0}^{\eta} \partial_{\eta} v_{2}^{m}(s, \tau) \mathrm{d} s\right) \text { o } \Phi^{-1}, \text { in } \mathcal{O}^{m}
\end{array}\right.
$$

Then, we have

$$
\left\|V-V_{a p p}\right\|_{L^{2}(\Omega)}=O\left(\delta^{3 / 2-\alpha}\right), \quad \text { uniformly for } \delta \text { tending to zero. }
$$

Remark 5.2. Since $\alpha$ belongs to $[0,1 / 2)$, Theorem 5.1 combined with Lemma 4.1 leads obviously to Theorem 1.1.

If the curve $\Gamma_{\delta}^{r}$ has no oscillating part, which means that $\alpha=0$, the above function $v_{1}$ and $\overline{v_{1}}$ are equal. And the above theorem with $\alpha=0$ is exactly Theorem 1.2.

Proof. It is convenient to set

$$
v_{a p p}^{m}=v_{0}^{m}+\delta v_{1}^{m}+\delta^{2} \int_{0}^{\eta} \partial_{\eta} v_{2}^{m}(s, \tau) \mathrm{d} s .
$$

Observe that

$$
\partial_{\eta} v_{a p p}^{m}(\eta, \theta)=\left.\delta f\left(\theta, \theta / \delta^{\alpha}\right) \frac{\sigma_{i}}{\sigma_{m}} \partial_{n} v_{0}^{i}\right|_{\Gamma^{-}} \mathrm{o} \Psi+\delta^{2} \partial_{\eta} v_{2}^{m}(\eta, \theta)
$$

Define

$$
W=\frac{V_{\delta}-V_{a p p}}{\delta}
$$

In each domain $\mathcal{O}_{\delta}^{e}$ and $\mathcal{O}^{i}, W$ is a harmonic function. In $\mathcal{O}_{\delta}$, according to (18) and the definition of $V_{a p p}$ we have:

$$
\begin{aligned}
\Delta W & =\frac{\delta^{2}}{|G|}\left\{\kappa(\theta) f(\tau)\left(2 \eta \partial_{\eta}^{2} v_{2}^{m}+\partial_{\eta} v_{2}^{m}\right)+\delta\left\{\left(\eta^{2}\left(\nabla_{\alpha} \cdot f(\tau)\right)^{2}\right.\right.\right. \\
& \left.+(\eta \kappa(\theta) f(\tau))^{2}\right) \partial_{\eta}^{2} v_{2}^{m}+f^{2}(\tau) \partial_{\theta}^{2} v_{2}^{m} \\
& -2 \eta f(\tau) \nabla_{\alpha} \cdot f(\tau) \partial_{\theta} \partial_{\eta} v_{2}^{m}+\eta\left(\kappa^{2}(\theta) f^{2}(\tau)+2\left(\nabla_{\alpha} \cdot f(\tau)\right)^{2}\right. \\
& \left.\left.\left.-f(\tau) \nabla_{\alpha}^{2} \cdot f(\tau)\right) \partial_{\eta} v_{2}^{m}\right\}+R(\eta, \tau) v_{a p p}^{m}\right\} \mathrm{o} \Phi^{-1},
\end{aligned}
$$

According to (19):

$$
\begin{aligned}
R(\eta, \tau) v_{a p p}^{m} & =\frac{\eta f(\tau)}{1+\kappa(\theta) \eta \delta f(\tau)}\left\{\eta\left(\kappa^{\prime}(\theta) \nabla_{\alpha} f-f^{2}(\tau) \kappa^{3}(\theta)\right) \partial_{\eta} v_{a p p}^{m}\right. \\
& \left.-\kappa^{\prime}(\theta) f^{2}(\tau) \partial_{\theta} v_{a p p}^{m}\right\} .
\end{aligned}
$$

Denote by $\gamma_{\circ} \Phi^{-1}$ the right hand side of (27). Using Lemma 3.1 we infer:

$$
\sup _{\eta \in[0,1]}|\gamma|_{H^{-1 / 2}(\mathbb{T})} \leq C \delta^{-\alpha}|\varphi|_{H^{7 / 2}(\partial \Omega)}
$$


We have for all $\phi \in H^{1}\left(\mathcal{O}_{\delta}\right)$,

$$
\begin{aligned}
\left|\int_{\mathcal{O}_{\delta}} \gamma \circ \Phi^{-1}(x) \phi(x) \mathrm{d} x\right| & =\left|\int_{0}^{1} \int_{0}^{2 \pi} \sqrt{|G|} \gamma(\eta, \theta) \phi \circ \Phi(\eta, \theta) \mathrm{d} \eta \mathrm{d} \theta\right| \\
& \leq C \delta^{1 / 2} \sup _{\eta \in[0,1]}|\gamma|_{H^{-1 / 2}(\mathbb{T})}\|\phi\|_{H^{1}\left(\mathcal{O}_{\delta}\right)} \\
& \leq C \delta^{1 / 2-\alpha}|\varphi|_{H^{7 / 2}(\partial \Omega)} \cdot\|\phi\|_{H^{1}\left(\mathcal{O}_{\delta}\right)}
\end{aligned}
$$

Observe that $W$ satisfies the following transmission conditions on $\Gamma$ :

$$
\begin{aligned}
& \left.W\right|_{\Gamma^{+}}-\left.W\right|_{\Gamma^{-}}=0, \\
& \left.\sigma_{m} \partial_{n} W\right|_{\Gamma^{+}}-\left.\sigma_{i} \partial_{n} W\right|_{\Gamma^{-}}=0,
\end{aligned}
$$

and on $\Gamma_{\delta}^{r}$,

$$
\begin{aligned}
& \left.W\right|_{\Gamma_{\delta}^{r+}}-\left.W\right|_{\Gamma_{\delta}^{r-}}=\frac{1}{\delta}\left(\left.V_{a p p}\right|_{\Gamma_{\delta}^{r-}}-\left.V_{a p p}\right|_{\Gamma_{\delta}^{r+}}\right), \\
& \left.\sigma_{e} \partial_{n} W\right|_{\Gamma_{\delta}^{r+}}-\left.\sigma_{m} \partial_{n} W\right|_{\Gamma_{\delta}^{r-}}=\frac{1}{\delta}\left(\left.\sigma_{m} \partial_{n} V_{a p p}\right|_{\Gamma_{\delta}^{r-}}-\left.\sigma_{e} \partial_{n} V_{a p p}\right|_{\Gamma_{\delta}^{r+}}\right) .
\end{aligned}
$$

Using a Taylor expansion with respect to the normal variable $\eta$, we explicit the terms $\left(\left.v_{j}^{e}\right|_{\Gamma_{\delta}^{r+}}\right)_{j=0,1}$ and $\left(\left.\partial_{n} v_{j}^{e}\right|_{\Gamma_{\delta}^{r+}}\right)_{j=0,1}$ in terms of $\left(\left.v_{j}^{e}\right|_{\Gamma^{+}}\right)_{j=0,1},\left(\left.\partial_{n} v_{j}^{e}\right|_{\Gamma^{+}}\right)_{j=0,1}$ and $\left(\left.\partial_{t} v_{j}^{e}\right|_{\Gamma^{+}}\right)_{j=0,1}$. Actually, in local coordinates $(\eta, \theta)$, we have:

$$
\begin{aligned}
& \left(\left.W\right|_{\Gamma_{\delta}^{r+}}-\left.W\right|_{\Gamma_{\delta}^{r-}}\right) \text { o }\left.\Phi\right|_{\eta=1}=\delta f\left(\theta, \theta / \delta^{\alpha}\right) \int_{0}^{1}(1-s)\left(\partial_{\eta}\left(v_{1}^{e} \circ \Phi\right)(s, \theta)\right. \\
& \left.+\frac{1-s}{2} f\left(\theta, \theta / \delta^{\alpha}\right) \partial_{\eta}^{2}\left(v_{0}^{e} \circ \Phi\right)(s, \theta)\right) \mathrm{d} s, \\
& \left(\left.\sigma_{e} \partial_{n} W\right|_{\Gamma_{\delta}^{r+}}-\left.\sigma_{m} \partial_{n} W\right|_{\Gamma_{\delta}^{r-}}\right) \text { o }\left.\Phi\right|_{\eta=1}=-\sigma_{e} \delta\left(\int _ { 0 } ^ { 1 } ( 1 - s ) f ( \tau ) \left(\partial_{n}^{2} v_{1}^{e}\right.\right. \\
& \left.+\frac{(1-s) f^{2}(\tau)}{2} \partial_{n}^{3} v_{0}^{e}\right) \text { o } \Phi(s, \theta) \mathrm{d} s \\
& -\nabla_{\alpha} \cdot f(\tau)\left(\left.\partial_{t} v_{1}^{e} \circ \Phi\right|_{\eta=1}\right. \\
& \left.+\int_{0}^{1} \partial_{n} \partial_{t} v_{0}^{e} \mathrm{o} \Phi \mathrm{d} s\right) \\
& +\nabla_{\alpha \cdot f}(\tau)\left(\left.\nabla_{\alpha \cdot f} f(\tau) \partial_{n}^{2} v_{0}^{e} \mathrm{o} \Phi\right|_{\eta=1}\right. \\
& \left.\left.+\left.\kappa(\theta) f(\tau) \partial_{t} v_{0}^{e} \mathrm{o} \Phi\right|_{\eta=1}\right)\right) \mathrm{d} s \\
& +\frac{\sigma_{m}}{f(\tau)}\left\{\nabla _ { \alpha \cdot f ( \tau ) } \left(\frac{\nabla_{\alpha} \cdot f(\tau)}{2} \partial_{\eta} v_{a p p}^{m}\right.\right. \\
& \left.\left.-f(\tau) \partial_{\theta} v_{a p p}^{m}\right)+T(\tau) v_{a p p}^{m}\right\}\left.\right|_{\eta=1}
\end{aligned}
$$


Observe that since $\Phi(1, \theta)=\Psi(\theta)+\delta f\left(\theta / \delta^{\alpha}\right) n(\theta)$, the following estimates hold

$$
\begin{aligned}
\left\|\left.W\right|_{\Gamma_{\delta}^{r+}}-\left.W\right|_{\Gamma_{\delta}^{r-}}\right\| H_{H^{1}\left(\Gamma_{\delta}^{r}\right)} \leq C_{\Gamma}\left(1+\delta^{1-\alpha}\right) \|\left(\left.W\right|_{\Gamma_{\delta}^{r+}}\right. & \\
& \left.-\left.W\right|_{\Gamma_{\delta}^{r-}}\right)\left.\circ \Phi\right|_{\eta=1} \|_{H^{1}(\mathbb{T})},
\end{aligned}
$$

and similarly

$$
\begin{aligned}
\left\|\left.\sigma_{e} \partial_{n} W\right|_{\Gamma_{\delta}^{r+}}-\left.\sigma_{m} \partial_{n} W\right|_{\Gamma_{\delta}^{r-}}\right\|_{L^{2}\left(\Gamma_{\delta}^{r}\right)} & \leq C_{\Gamma} \|\left(\left.\sigma_{e} \partial_{n} W\right|_{\Gamma_{\delta}^{r+}}\right. \\
& \left.-\left.\sigma_{m} \partial_{n} W\right|_{\Gamma_{\delta}^{r-}}\right)\left.\circ \Phi\right|_{\eta=1} \|_{L^{2}(\mathbb{T})} .
\end{aligned}
$$

Therefore, from the right-hand side of (28)-(29) and using the definitions of $\left(v_{j}^{e}\right)_{j=0,1}$ and $\left(v_{j}^{m}\right)_{j=0,1}$, we infer easily with the help of Lemma 3.1 that there exists two functions $b_{1}$ and $b_{2}$ :

$$
\begin{gathered}
\left\|b_{1}\right\|_{H^{1}\left(\Gamma_{\delta}^{r}\right)} \leq C|\varphi|_{H^{7 / 2}(\partial \Omega)}, \\
\left\|b_{2}\right\|_{L^{2}\left(\Gamma_{\delta}^{r}\right)} \leq C|\varphi|_{H^{7 / 2}(\partial \Omega)} .
\end{gathered}
$$

and

$$
\begin{aligned}
& \left.W\right|_{\Gamma_{\delta}^{r+}}-\left.W\right|_{\Gamma_{\delta}^{r-}}=\delta^{1-2 \alpha} b_{1}, \\
& \left.\sigma_{e} \partial_{n} W\right|_{\Gamma_{\delta}^{r+}}-\left.\sigma_{m} \partial_{n} W\right|_{\Gamma_{\delta}^{r-}}=\delta^{1-2 \alpha} b_{2} .
\end{aligned}
$$

Then we have shown that $W$ satisfies:

$$
\begin{aligned}
& \Delta W=0, \quad \text { in } \mathcal{O}_{\delta}^{e} \cup \mathcal{O}^{i}, \\
& \Delta W=\gamma \mathrm{o} \Phi^{-1}, \quad \text { in } \mathcal{O}_{\delta}, \\
& \left.W\right|_{\Gamma^{+}}-\left.W\right|_{\Gamma^{-}}=0, \\
& \left.\sigma_{m} \partial_{n} W\right|_{\Gamma^{+}}-\left.\sigma_{i} \partial_{n} W\right|_{\Gamma^{-}}=0, \\
& \left.W\right|_{\Gamma_{\delta}^{r+}}-\left.W\right|_{\Gamma_{\delta}^{r-}}=\delta^{1-2 \alpha} b_{1}, \\
& \left.\sigma_{e} \partial_{n} W\right|_{\Gamma_{\delta}^{r+}}-\left.\sigma_{m} \partial_{n} W\right|_{\Gamma_{\delta}^{r-}}=\delta^{1-2 \alpha} b_{2}, \\
& \left.W\right|_{\partial \Omega}=0 .
\end{aligned}
$$

For all $\psi \in L^{2}(\Omega)$, define $\phi \in H_{0}^{1}(\Omega)$ by:

$$
\nabla \cdot(\sigma \nabla \phi)=\psi, \quad \text { in } \Omega .
$$

It is well-known that

$$
\|\phi\|_{H^{1}(\Omega)} \leq C\|\psi\|_{L^{2}(\Omega)} .
$$

Moreover, since the amplitude of the oscillations of $\Gamma_{\delta}^{r}$ are the square of their period, the result of Bonder et al. [6] leads to the following estimates for an $\delta$-independent constant $C$ :

$$
\begin{aligned}
& |\phi|_{L^{2}\left(\Gamma_{\delta}^{r}\right)} \leq C\|\psi\|_{L^{2}(\Omega)}, \\
& \left|\partial_{n} \phi\right|_{H^{-1}\left(\Gamma_{\delta}^{r}\right)} \leq C\|\psi\|_{L^{2}(\Omega)} .
\end{aligned}
$$


Then, multiplying (30) by $\phi$ and integrating by parts, there exists a constant $C$ such that:

$$
\left|\int_{\Omega} W(x) \psi(x) \mathrm{d} x\right| \leq C \delta^{1 / 2-\alpha}|\varphi|_{H^{7 / 2}(\partial \Omega)}\|\psi\|_{L^{2}(\Omega)} .
$$

Hence we infer

$$
\|W\|_{L^{2}(\Omega)} \leq C \delta^{1 / 2-\alpha}|\varphi|_{H^{7 / 2}(\partial \Omega)} .
$$

Since $\alpha<1 / 2$, the theorem is shown.

\section{ACKNOWLEDGEMENTS}

The former author thanks very warmly S. Ciuperca and H. Ammari for their well-considered advice and suggestions.

\section{REFERENCES}

[1] Toufic Abboud and Habib Ammari. Diffraction at a curved grating: TM and TE cases, homogenization. J. Math. Anal. Appl., 202(3):995-1026, 1996.

[2] Yves Achdou and Olivier Pironneau. Domain decomposition and wall laws. C. R. Acad. Sci. Paris Sér. I Math., 320(5):541-547, 1995.

[3] Habib Ammari and Hyeonbae Kang. Properties of the generalized polarization tensors. Multiscale Model. Simul., 1(2):335-348 (electronic), 2003.

[4] Habib Ammari and Hyeonbae Kang. Reconstruction of conductivity inhomogeneities of small diameter via boundary measurements. In Inverse problems and spectral theory, volume 348 of Contemp. Math., pages 23-32. Amer. Math. Soc., Providence, RI, 2004.

[5] Elena Beretta, Elisa Francini, and Michael S. Vogelius. Asymptotic formulas for steady state voltage potentials in the presence of thin inhomogeneities. A rigorous error analysis. J. Math. Pures Appl. (9), 82(10):1277-1301, 2003.

[6] Julián Fernández Bonder, Rafael Orive, and Julio D. Rossi. The best Sobolev trace constant in a domain with oscillating boundary. Nonlinear Anal., 67(4):1173-1180, 2007.

[7] Yves Capdeboscq and Michael S. Vogelius. A general representation formula for boundary voltage perturbations caused by internal conductivity inhomogeneities of low volume fraction. M2AN Math. Model. Numer. Anal., 37(1):159-173, 2003.

[8] I. Ciuperca, M. Jai, and Poignard C. Approximated transmission conditions for a highly oscillating thin layer. In preparation.

[9] B. A. Dubrovin, A. T. Fomenko, and S. P. Novikov. Modern geometry-methods and applications. Part I, volume 93 of Graduate Texts in Mathematics. Springer-Verlag, New York, second edition, 1992. The geometry of surfaces, transformation groups, and fields, Translated from the Russian by Robert G. Burns.

[10] E.C. Fear and M.A. Stuchly. Modeling assemblies of biological cells exposed to electric fields. IEEE Trans.Bio.Eng, 45(1):1259-1271 (electronic), 1998.

[11] K.R. Foster and H.P. Schwan. Dielectric properties of tissues and biological materials: a critical review. CRC in Biomedical Engineering, 17(1):25-104, 1989.

[12] Alexandre Madureira and Frédéric Valentin. Analysis of curvature influence on effective boundary conditions. C. R. Math. Acad. Sci. Paris, 335(5):499-504, 2002.

[13] S. Muñoz, J.L. Sebastián, M. Sancho, and J.M. Miranda. Transmembrane voltage induced on altered erythrocyte shapes exposed to rf fields. Bioelectromagnetics, 25(1):631-633 (electronic), 2004.

[14] C. Poignard. Méthodes asymptotiques pour le calcul de champs électromagnétiques dans des milieux à couche mince. Application aux cellules biologiques. Thesis, November 2006.

[15] C. Poignard, P. Dular, R. Perrussel, L. Krähenbühl, L. Nicolas, and M. Schatzman. Approximate condition replacing thin layer. IEEE Trans. on Mag., 2007. To appear. http://hal.archives-ouvertes.fr/hal-00165049/fr.

[16] Clair Poignard. Asymptotics for steady state voltage potentials in a bidimensionnal highly contrasted medium with thin layer. To appear in Math. Meth. Appl. Sci. http://www.cmap.polytechnique.fr/ poignard/MMAS-31pp.pdf, 2007. 
[17] G Pucihar, T Kotnik, B Valič, and D Miklavčič. Numerical determination of transmembrane voltage induced on irregularly shaped cells. Annals of Biomedical Engineering, 34(4):642-652 (electronic), 2006.

[18] J.L. Sebastián, S. Muñoz, M. Sancho, and J.M. Miranda. Analysis of the influence of the cell geometry and cell proximity effects on the electric field distribution from direct rf exposure. Phys. Med. Biol., 46:213-225 (electronic), 2001.

[19] D. Sel, D. Cukjati, D. Batiuskaite, T. Slivnik, L.M. Mir, and D. Miklavcic. Sequential finite element model of tissue electropermeabilization. IEEE Trans. Bio. Eng., 52(5):816-827, 2005.

[20] T.Y. Tsong. Electroporation of cell membranes. Biophys J., 60:297-306, 1991.

Laboratoire Ondes et Acoustique, CNRS \& ESPCI, 10 rue Vauquelin, 75231 Paris Cedex 05, France., mailto: Poignard@CMAPX.POlytechnique.Fr 